\title{
ADVERSE DRUG REACTION WITH HYOSCINE AND VALETHAMATE FOR CERVICAL DILATION DURING LABOUR
}

\author{
Tuladhar LR $R^{1}$, Shrestha $A^{1}$, Shrestha $R K^{2}$
}

${ }^{1}$ Department of Pharmacology, Nepal Medical College Teaching Hospital, Attarkhel, Gokarneshwor-8, Kathmandu, ${ }^{2}$ Patan Academy of Health Sciences (PAHS), Patan, Nepal.

\begin{abstract}
Adverse drug reaction (ADR) is an injury caused by taking medication. ADR may occur following single dose or prolong administration of drug or combination of two or more drugs. While major advancements of discipline of pharmacovigilance have taken place in the West, not much has been achieved in Asian countries. Labour is characterised by forceful and painful uterine contraction that result in cervical dilation and foetus decent from the birth canal. Anti-spasmodic drugs like hyoscine butylbromide and valethamate bromide have been used to accelerate cervical dilation and thus reduce the labor duration. The objective was to observe ADR with hyoscine and valethamate for cervical dilation during labor. It was a hospital based cross sectional study. Investigation was carried out in the form of questionnaire. All the consecutive patients who were in active stage of labor were included in the study. They were given Intravenous (IV) valethamate bromide 8mg and hyoscine butylbromide 20mg, 3 doses half an hour apart. After administration of the drug, the progress of labor was monitored and management was done as per protocol in obstetrics and gynaecology department. ADR reported were blurred vision in $47.7 \%$ of the patients, followed by dry mouth (36.9\%) and tachycardia (19.2\%). Other ADRs were nausea $(6.2 \%)$, dizziness (3.8\%), flushing (2.3\%), vomiting (1.5\%), fever (1.5\%) and constipation (1.5\%). No statistical significance was found when ADR was compared between the age group of 18-25 years and 26-35 years. Therefore, ADR reported were irrespective of the age of the patients and no life threatening or severe forms of ADR were seen with hyoscine and valethamate during cervical dilation.
\end{abstract}

\section{KEYWORDS}

Adverse drug reaction, hyoscine butylbromide, valethamate bromide, cervical dilation, labor

\section{CORRESPONDING AUTHOR}

\author{
Dr. Lujaw Ratna Tuladhar, \\ Lecturer, \\ Department of Pharmacology, \\ Nepal Medical College Teaching Hospital, \\ Attarkhel, Gokarneshwor-8, Kathmandu, Nepal, \\ Email: lujaw3@gmail.com
}




\section{INTRODUCTION}

Adverse drug reaction (ADR) is injuries that occurs following single dose or prolong administration of drug or combination of them. The study of ADR is called pharmacovigilance whose market is expected to reach USD 10.27 billion by 2025 and witness growth at $13.1 \% .{ }^{1}$ Increasing incidence of ADR is key driver for growth of pharmacovigilance market. While major advancements of discipline of pharmacovigilance have taken place in the west, not much have been achieved in Asian countries and remains a constant challenge. ${ }^{2}$ This study is an effort to monitor ADR for drugs used for cervical dilation.

Of all the journeys ever we make, the most dangerous one is the first one we take through the last $10 \mathrm{~cm}$ of the birth canal. ${ }^{1}$ Can drug administration further increase this risk?

Labor is the process of childbirth. Labor has three stages. ${ }^{3}$ During the first stage, the muscle of uterus contract and soften the cervix so that it can dilate upto $10 \mathrm{~cm} .{ }^{4}$ During the second stage: the baby moves down through the vagina and is born. ${ }^{5}$ During the third stage, the placenta is delivered. ${ }^{6}$ Prolonged labor contribute to increased perinatal and maternal morbidity. ${ }^{7,8}$ This can be avoided by use of pharmacological preparation that help to reduce duration of labor. ${ }^{9}$ Painless and short labor has been a desire for every women and goal of every obstetrician that results in healthy and minimally traumatized mother. Cervical dilation is one of the important factor which determine the duration of labor. ${ }^{10,11}$ Another factor that prolong labour is pain sensation..$^{12}$ Prolong labor however leads to maternal complication like endometritis, post-partum haemorrhage and fetal complications. ${ }^{13}$ Stimulation of sympathetic chain cause contraction of uterus whereas stimulation of parasympathetic chain cause contraction of cervix. ${ }^{14}$

Anti-spasmodic drugs have been used to accelerate cervical dilation and thus reduce the labor duration. ${ }^{15,16}$ Valethamate bromide injection is an anti-spasmodic agent, and is used in treating dysmenorrhea, post-operative pain and spasms. Valethamate bromide has neurotropic and musculotropic action, resulting in relaxation of cervical musculature leading to quick dilation of cervix and shortened labour. Valethamate bromide 8 $\mathrm{mg}$ can be injected intravenously or intramuscularly as required..$^{17,18}$

Hyoscine butylbromide is an anti-cholinergic drug that inhibiting cholinergic transmission in the synapse of the abdominal and pelvic parasympathetic ganglia, thus relieving spasm in the smooth muscle of female genital organ especially the cervico uterine plexus thus aiding cervical dilation. Hyoscine is a medication used to treat crampy abdominal pain, esophageal spasms, renal colic, and bladder spasms. It has selective action on cervix and accelerates labour by dilating cervix. Hyoscine can be delivered by injecting into a vein. ${ }^{19,20}$
The combination of Valethamate bromide and Hyoscine butylbromide is given in the first stage of labor to facilitate the cervical dilation. ${ }^{21}$ After administration of the drug within therapeutic dose, a number of unwanted and undesirable effects have been observed which is termed as adverse drug reaction. Studies have reported adverse drug reaction like vomiting in $10 \%$, dryness of mouth in $10 \%$ and tachycardia in 3\%. ${ }^{22}$

\section{MATERIALS AND METHODS}

It is a hospital based cross sectional study conducted in department of obstetrics and gynaecology, Nepal Medical College and Teaching Hospital (NMCTH). The protocol was reviewed and approved by Institutional Review Committee (IRC) of NMCTH. Informed consent was taken from patients. A validated questionnaire was distributed that contained a list of ADR of Hyoscine and Valethamate and the duty doctor was instructed to fill the questionnaire. All the consecutive patients who were in active stage of labor (cervical dilation ranging from $4 \mathrm{~cm}-9 \mathrm{~cm}$ ) were included in the study. They were given slow IV inj Valethamate bromide $8 \mathrm{mg}$ and Hyoscine butylbromide 20mg mixed together in the same syringe, 3 doses half an hour apart by the nursing staff. After administration of the drug, the progress of labor was monitored and management was done as per protocol in obstetrics and gynaecology department. Patient with history of allergy, liver, kidney and cardiac disease were excluded from the study.The data were collected from the period of $4^{\text {th }}$ August 2017 till 23 ${ }^{\text {rd }}$ September 2018. Data were entered in SPSS version 16 and chi square test (Pearson chi square and fisher exact test) was applied. P value $<0.05$ was considered as significant.

\section{RESULTS}

A total of 130 pregnant women in the labor ward preparing for delivery were enrolled in this study. Data were taken from patient receiving Hyoscine (Buscopan) and Valethamate (Epidosin). Patients were from the age group of 18-35 years. The mean age was $25.5 \pm 4.36$ years.

After slow IV administration of Valethamate $8 \mathrm{mg}$ and Hyoscine 20mg combined together in the same syringe to the patient, we observed ADR like blurred vision, dry mouth, tachycardia, nausea, dizziness, flushing, vomiting, fever and constipation. The most frequent ADR was blurred vision in $47.7 \%$ of the patients, followed by dry mouth $(36.9 \%)$ and tachycardia (19.2\%). Other ADR were nausea (6.2\%), dizziness $(3.8 \%)$, flushing (2.3\%), vomiting (1.5\%), fever (1.5\%) and constipation (1.5\%). No statistical significance was found when ADR was compared between the age group of 18-25 years and 26-35 years. Therefore, ADR reported were irrespective of the age of the patients. No life threatening ADR were reported. 
Table 1: Frequency of Adverse Drug Reaction reported for hyoscine and valethamate.

\begin{tabular}{|lcc|} 
& $\mathbf{n}$ & $\mathbf{\%}$ \\
Blurred vision & 62 & 47.70 \\
Dry mouth & 48 & 36.90 \\
Tachycardia & 25 & 19.20 \\
Nausea & 8 & 6.20 \\
Dizziness & 5 & 3.80 \\
Flushing & 3 & 2.30 \\
Vomiting & 2 & 1.50 \\
Fever & 2 & 1.50 \\
Constipation & 2 & 1.50 \\
Total & $\mathbf{1 3 0}$ & $\mathbf{1 0 0}$ \\
\hline
\end{tabular}

a large sample size of 130 pregnant female patients from the age group of 18-35 years. The mean age was 25.5 years that indicate healthy population. Hyoscine and Valethamate was combined in the same syringe that was delivered slow IV to produce cervical dilation and shorten labour duration.

The study revealed ADR like blurred vision, dry mouth, tachycardia, nausea, dizziness, flushing, vomiting, fever and constipation but no life threatening ADR were reported. Although ADR have been reported Changede et al reported that rate of cervical dilation under the influence of Valethamate and Hyoscine was significantly higher as compared to the control group concluding higher benefit over the risk. ${ }^{23}$

The most frequent ADR reported was blurring of vision that was seen in $47.7 \%$ of the patients due to anti-cholinergic action ${ }^{24}$ that produce relaxation of

\section{Table 2: Variation and frequency of Adverse Drug Reaction on the basis of age}

\begin{tabular}{|c|c|c|c|c|c|}
\hline & \multicolumn{2}{|c|}{$\leq 25$ years $(n=68)$} & \multicolumn{2}{|c|}{$>25$ years $(n=62)$} & \multirow{2}{*}{$p$ value } \\
\hline & $\mathrm{n}$ & $\%$ & $\mathbf{n}$ & $\%$ & \\
\hline Tachycardia & 13 & 19.1 & 12 & 19.7 & 0.937 \\
\hline Blurred vision & 30 & 44.1 & 32 & 52.5 & 0.344 \\
\hline Vomiting & 1 & 1.5 & 1 & 1.6 & $0.938 *$ \\
\hline Dizziness & 3 & 4.4 & 2 & 3.3 & $0.739 *$ \\
\hline Dry mouth & 25 & 36.8 & 22 & 36.1 & 0.934 \\
\hline Fever & 1 & 1.5 & 1 & 1.6 & $0.938 *$ \\
\hline Constipation & 2 & 2.9 & 0 & 0.0 & $0.177^{*}$ \\
\hline Flushing & 2 & 2.9 & 1 & 1.6 & $0.624^{*}$ \\
\hline Nausea & 6 & 8.8 & 2 & 3.3 & $0.192 *$ \\
\hline
\end{tabular}

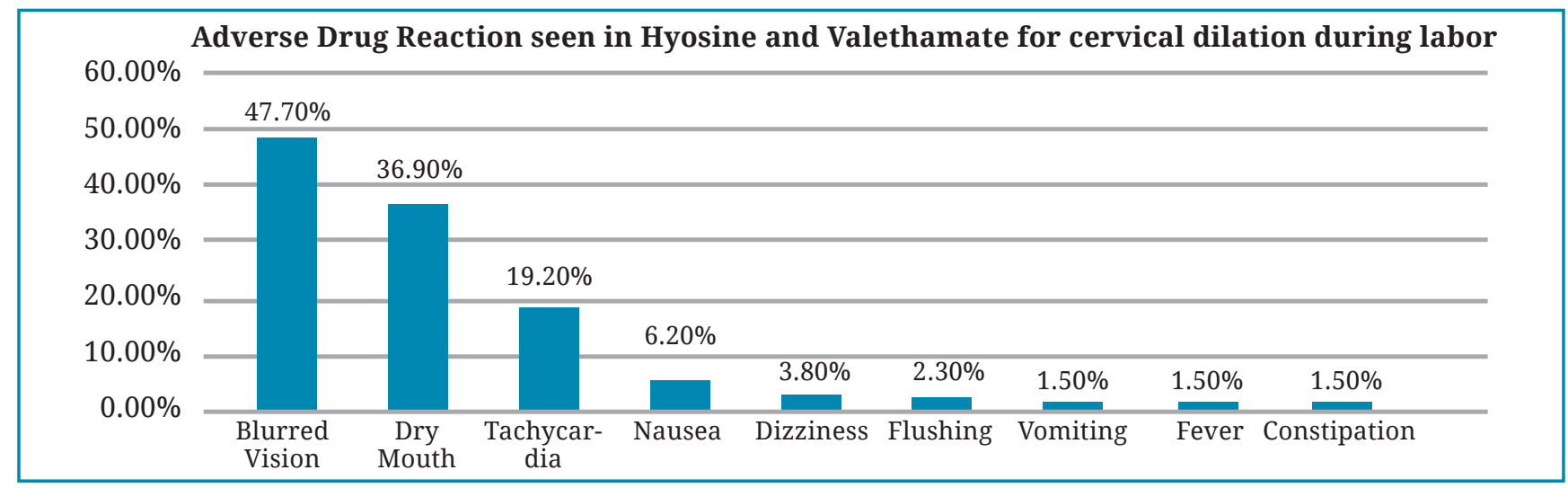

Fig.1: Adverse Drug Reaction seen in hyoscine and valethamate for cervical dilation during labor.

\section{DISCUSSION}

Adverse drug reaction (ADR) is a broad term referring to unwanted, unavoidable and uncomfortable effect following single dose or prolong administration of drug or combination of two or more drugs. ${ }^{2}$ This observational study is an effort to monitor ADR for drugs like Hyoscine and Valethamate. We recruited ciliary muscle by blocking muscarinic(M) receptor leading to cycloplegia. Since accommodation is totally dependent on parasympathetic innervations any form of blockade leads to blurring of vision..$^{25}$

The second most frequent ADR reported in our study were dry mouth in $36.9 \%$ followed by tachycardia in $19.2 \%$. This is also associated with anti-cholinergic 
action (decreased secretion, produces tachycardia, mydriasis, hyperthermia (resulting in vasodilation), sedation, urinary retention, constipation, behavioural excitation and hallucination) and therefore ADR can be predicted. ${ }^{26}$

Some studies have reported vomiting as the main ADR followed by dryness of mouth, nausea and transient tachycardia. Kaur et $a l^{20}$ noted dryness of mouth as the main side effect followed by vomiting $(6 \%)$, nausea (5\%), drowsiness (1\%), and transient tachycardia (1\%). Devinder et a ${ }^{27}$ and Kholsa et a ${ }^{28}$ in their study noted maternal tachycardia in $28 \%$ of cases and 16\% cases respectively. Therefore studies have shown similar ADR with variation only in their prevalence.

Flushing could be due to superficial vasodilation. ${ }^{29}$ Anti-cholinergic drugs block sweating, this results in overheating and compensatory superficial cutaneous vasodilation to increase heat loss.

Sharma et a $3^{30}$ noted that Valethamate bromide was effective in acceleration of labor with mild ADR. However some studies have questioned the use of Valethamate bromide during pregnancy as its efficacy has not been well documented. ${ }^{31}$ Kurucila et al concluded that there was no difference observed in the rate of cervical dilatation between those who received Valethamate bromide and those who received normal saline. However, maternal tachycardia was observed significantly more who had received Valethamate bromide than normal saline. ${ }^{32}$

Gitanjali reported that it was interesting to note that both saline and water have Indian Pharmacopoeia (I.P.) listed next to their names but what is missed by the undiscerning mind is that there is no I.P next to Valethamate bromide which itself is a question of safety and efficacy of Valethamate. The study

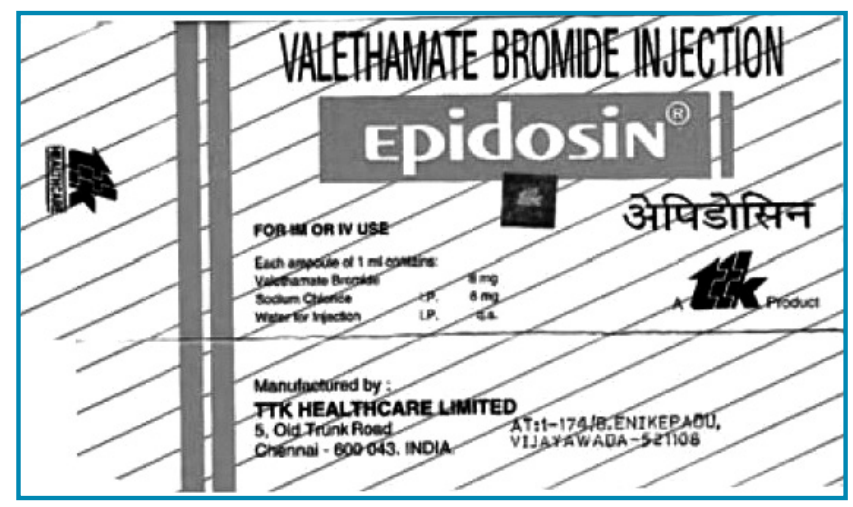

Fig. 2: A Photograph of the carton of Epidosin (valethamate bromide)

also looks forward for the answer as Valethamate does not satisfy even one of the WHO guideline for selection of drug for any indication. ${ }^{31}$

Similarly Hyoscine also shortens the duration of labor in pregnancy with no ADR reported. ${ }^{33}$ Another study by Aggarwal et al concluded that
Hyoscine produced pain relief of up to $35.6 \%$ with no adverse maternal complications. Pain relief of $35.6 \%$ was noted on visual analog score with the use of Hyoscine. ${ }^{34}$ Similarly, Samuels et al, Makvandi et al, Kaur et al and Sekhavat et al in their studies reported no significant adverse outcome with Hyoscine in mother and fetus. ${ }^{19,20,35,36}$ Therefore ADR reported in our study may not be associated with Hyoscine either. Other ADR of anti-cholinergic group are cardiac toxicity, convulsion and coma ${ }^{37,38}$ however, there were not reported for Valethamate and Hyoscine because it is impermeable to blood brain barrier.

WHO Assistant Director-General for Family, Women, Children and Adolescents, Dr. Princess Nothemba Simelela said "We want women to give birth in a safe environment with skilled birth attendants in well-equipped facilities. However, the increasing medicalization of normal childbirth processes are undermining a woman's own capability to give birth and negatively impacting her birth experience. If labour is progressing normally, and the woman and her baby are in good condition, they do not need to receive additional interventions to accelerate labour." 39

Similarly WHO Director, Department of Reproductive Health and Research, Ian Askew said "Many women want a natural birth and prefer to rely on their bodies to give birth to their baby without the aid of medical intervention. Even when a medical intervention is wanted or needed, the inclusion of women in making decisions about the care they receive is important to ensure that they meet their goal of a positive childbirth experience.”39 Therefore, medical intervention was not advised by WHO unless needed.

Our study could not identify the key drug for ADR. This is because, in practice, Hyoscine and Valethamate were combined together when used. Some studies have reported Valethamate to be safe without major ADR. Therefore, it seems that reported ADR could be associated with Hyoscine. ${ }^{40}$ Whereas Some researchers have concluded that Hyoscine is not associated with any apparent adverse maternal or neonatal outcome. ${ }^{41,19}$ Therefore, it is unlikely that the ADR reported in our study is associated only with Hyoscine.

Therefore, it is possible that the ADR reported could be due to combination of Hyoscine and Valethamate both that might produce supra-additive outcome with minimalistic ADR as reported in our study. For ADR associated with individual drug individual study on Hyoscine, Valethamate would be required.

The purpose of the study was to identify ADR with IV Hyoscine-8mg and Valethamate-20mg combination when used for cervical dilation. ADR reported were expected and could not be linked to maternal complication considering greater benefit over the risk. Hence, we conclude combination of Valethamate and Hyoscine as safe. 


\section{REFERENCES}

1. Wiktorowicz M, Lexchin J, Moscou K. Pharmacovigilance in Europe and North America: Divergent approaches. Soc Sci Med 2012; 75: 16570.

2. Biswas P. Pharmacovigilance in Asia. J Pharmacol Pharmacother 2013; 4: s7-19.

3. Dixon L, Skinner J, Foureur M. Women's perspectives of the stages and phases of labour. Midwifery 2013; 29: 10-7.

4. Lawrence A, Lewis L, Hofmeyr GJ, Dowswell T, Styles C. Maternal positions and mobility during first stage labour. Sao Paulo Med J 2011; 129: 362-5.

5. Holvey N. Supporting women in the second stage of labour. Br J Midwifery 2014; 22: 182-6.

6. Davies L. Physiological third stage -- how long is too long? Essentially MIDIRS 2011; 2: 38-9.

7. Pennell CE, Henderson JJ, O’Neill MJ, McCleery S, Doherty DA, Dickinson JE. Induction of labour in nulliparous women with an unfavourable cervix: A randomised controlled trial comparing double and single balloon catheters and PGE2gel. BJOG: An Int'l J Obstet Gynaecol 2009; 116: 1443-52.

8. Lavender T, Hart A, Walkinshaw S, Campbell E, Alfirevic Z. Progress of first stage of labour for multiparous women: An observational study. BJOG: An Int'l J Obstet Gynaecol 2005; 112: 1663-5.

9. Trout KK. The neuromatrix theory of pain: Implications for selected nonpharmacologic methods of pain relief for labor. J Midwifery Women's Health 2004; 49: 482-8.

10. Lin MG, Rouse DJ. What is a failed labor induction? Clin Obstet Gynecol 2006; 49: 585-93.

11. Adams J, Frawley J, Steel A, Broom A, Sibbritt D. Use of pharmacological and non-pharmacological labour pain management techniques and their relationship to maternal and infant birth outcomes: Examination of a nationally representative sample of 1835 pregnant women. Midwifery 2015; 31: 45863.

12. Lally JE, Thomson RG, MacPhail S, Exley C. Pain relief in labour: a qualitative study to determine how to support women to make decisions about pain relief in labour. BMC Pregnancy Childbirth 2014; 14: 6-16.

13. Movahed F, Ghaleh TD, Lalooha F. Effect of Hyoscine on First Stage of Labour in Full Term Pregnancy. Res J Biol Sci 2011; 6: 648-51.

14. Arnot PH. Prolonged labor. Calif Med 1952; 76: 202 .

15. Sirohiwal D, Dahiya K, De M. Efficacy of hyoscineN-butyl bromide (Buscopan) suppositories as a cervical spasmolytic agent in labour. Aust New Zeal J Obstet Gynaecol 2005; 45: 128-9.
16. Madhu C, Mahavarkar S, Bhave S. A randomised controlled study comparing Drotaverine hydrochloride and Valethamate bromide in the augmentation of labour. Arch Gynecol Obstet 2010; 282: 11-5.

17. Srujana R, Shailaja N, Krishna L, Kulkarni N, Kaur $\mathrm{P}$, Bhat BS. To compare and evaluate the efficacy and safety of drotaverine, valethamate bromide and the combination of both the drugs. Biomed 2013; 33: 318-24.

18. Sreelatha S VN. Effect of Valethamate Bromide on the First Stage of Labor. Indian J Clin Pract 2013; 24: $166-7$.

19. Sekhavat L, Karbasi SA, Fallah R, Mirowliai M. Effect of hyoscine butylbromide first stage of labour in multiparus women. Afr Health Sci 2012; 12: 408-11.

20. Sarbhjit K, Bajwa SK, Parmjit K, Surinder B. To compare the effect of camylofin dihydrochloride (Anafortin) with combination of valethamate bromide (epidosin) and hyoscine butyl-n-bormide (buscopan) on cervical dilation. J Clin Diagnostic Res 2013; 7: 1897-9.

21. Jayashree S, Ajjammanavar V, Sujatha M. Comparison of Drotaverine and Valethamate Bromide In First Stage Of Labor. Int'l J Biol Med Res 2013; 4: 3215-8.

22. SarbhjitK. ResearchandReviews :JournalofMedical and Health Sciences To Evaluate the Incidence of Side Effects of Camylofindihydrochloride with ( Buscopan ), on Mother and to Look for Neonatal Outcome . Res Rev Med Health Sci 2013; 2: 33-7.

23. Changede PR. Comparison of injection drotaverine and injection valethamate bromide on duration and course of labor. Int'l J Reprod Contraception, Obstet Gynecol 2016; 5: 1836-42.

24. Mitchelson F. Muscarinic receptor agonists and antagonists: effects on ocular function. Handb Exp Pharmacol 2012; 208: 263-98.

25. Richelson E. Antimuscarinic and other receptorblocking properties of antidepressants. Mayo Clin Proc 1983; 58: 40-6.

26. Feinberg $M$. The problems of anticholinergic adverse effects in older patients. Drugs Aging 1993; 3: $335-48$.

27. Kaur D, Ravinder K. Comparison of Drotaverine and Epidosin in First stage of Labor. J Obs Ind 2003; 53: 449-52.

28. Khosla A, Bala I, Dahiya K. A comparative study of the efficacy of valethamate bromide with drotverine in normal labour. J Obs Ind 2003; 53: 568-70.

29. Sadeghian A, Rouhana H, Oswald-Stumpf B, Boh E. 
Etiologies and management of cutaneous flushing. J Am Acad Dermatol 2017; 77: 405-14.

30. Sharma JB, Pundir P, Kumar A, Murthy NS. Drotaverine hydrochloride vs. valethamate bromide in acceleration of labor. Int'l J Gynaecol Obstet 2001; 74: 255-60.

31. Gitanjali B. Valethamate bromide: Is there any proof of efficacy and safety for its use in labor? J Pharmacol Pharmacother 2010; 1: 2-3.

32. Kuruvila S, Jasper P, Peedicayil A, Mathai M. A randomized controlled trial of valethamate bromide in acceleration of labor. Int'l J Gynecol Obstet 1992; 38: 93-6.

33. Treviño-Salinas EM, Castillo-Martín del Campo GE, Ayuzo-del Valle C. Effect of hyoscine butylbromide on cervical dilation during labor. Med Univ 2015; 17: $30-3$.

34. Aggarwal P, Zutshi V, Batra S. Role of hyoscine N-butyl bromide (HBB, buscopan) as labor analgesic. Indian J Med Sci 2008; 62: 179-84.

35. Samuels LA, Christie L, Roberts-Gittens B. The effect of hyoscine butylbromide on the first stage of labour in term pregnancies. BJOG An Int'l J Obstet Gynaecol 2007; 114: 1542-6.
36. Makvandi S, Tadayon M, Abbaspour M. Effect of hyoscine-N-butyl bromide rectal suppository on labor progress in primigravid women: a randomized double-blind placebo-controlled clinical trial. Croat Med J 2011; 52: 159-63.

37. SiddaRama R, Baig MJ, Kumar AA. A case report on Atropine induced CNS side effects and Tachycardia. Int'l J Allied Med Sci Clin Res 2015; 3: 79-81.

38. Corallo CE, Whitfield A, Wu A. Anticholinergic syndrome following an unintentional overdose of scopolamine. Ther Clin Risk Mgmt 2009; 5: 719-23.

39. WHO. Individualized, supportive care key to positive childbirth experience, says WHO. WHO. 2018.

40. Dwivedi A, Rai G, Sharda S. Valethamate bromide and cervical dilatation. Med J Armed Forces India 1999; 55: 201-2.

41. Qahtani A, Nourah H, Fatma A. The effect of hyoscine butylbromide in shortening the first stage of labor: A double blind, randomized, controlled, clinical trial. Ther Clin Risk Mgmt 2011; 7: 495-500. 